\title{
Effects and potential mechanism of atorvastatin treatment on Lp-PLA2 in rats with dyslipidemia
}

\author{
Dongdan Zheng ${ }^{1}$, Anping $\mathrm{Cai}^{2}$, Rulin $\mathrm{Xu}^{1}$, Zhuocheng $\mathrm{Mai}^{3}$, Yingling Zhou ${ }^{2}$, Fanfang Zeng ${ }^{1}$, \\ Liwen $\mathrm{Li}^{2}$, Weiyi Mai ${ }^{1}$
}

\begin{abstract}
${ }^{1}$ Department of Cardiology, the First Affiliated Hospital of Sun Yat-sen University, Key Laboratory on Assisted Circulation, Ministry of Health, Guangzhou, China 2Department of Cardiology, Guangdong Cardiovascular Institute, Guangdong Provincial Key Laboratory of Coronary Heart Disease Prevention, Guangdong General Hospital, Guangdong Academy of Medical Sciences, Guangzhou, China

${ }^{3}$ Division of Biomedical Engineering, School of Engineering, Sun Yat-sen University, Guangzhou, China
\end{abstract}

Submitted: 10 July 2017

Accepted: 21 July 2017

Arch Med Sci 2018; 14, 3: 629-634

DOI: https://doi.org/10.5114/aoms.2017.69494

Copyright @ 2017 Termedia \& Banach

\section{Abstract}

Introduction: The effects of statins on lipoprotein-associated phospholipase A2 (Lp-PLA2) are controversial, and the present study aimed to investigate whether atorvastatin could reduce LP-PLA2 in rats with dyslipidemia.

Material and methods: A high-fat and high-cholesterol diet was prescribed to produce a dyslipidemia model. Thereafter, low-dose atorvastatin $(5 \mathrm{mg} /$ $\mathrm{kg} /$ day), high-dose atorvastatin $(20 \mathrm{mg} / \mathrm{kg} /$ day) or saline (without-treatment group) was prescribed for 14 days. At 6 weeks after dyslipidemia model establishment and 14 days of atorvastatin treatment, fasting venous blood was drawn for biochemical analysis. Between-group differences and Pearson correlation analysis were conducted.

Results: Compared to the normal-control group, fasting plasma total cholesterol (TC) and low-density lipoprotein cholesterol (LDL-C) levels were significantly increased in dyslipidemia groups, while plasma nitric oxide (NO) levels were significantly decreased with attendant elevation of plasma C-reactive protein (CRP) and rho-associated kinase 1 (ROCK1) levels $(p<0.05)$. At 14 days of atorvastatin treatment, compared to the without-treatment group, plasma levels of TC and LDL-C in the high-dose group were significantly reduced $(p<0.05)$; and compared to low-dose and without-treatment groups, NO up-regulation $(1.8 \pm 1.1 \mu \mathrm{mol} / \mathrm{l})$, and CRP $(-0.8 \pm 0.4 \mathrm{ng} / \mathrm{ml})$, ROCK1 $(-124$ $\pm 65 \mathrm{mmol} / \mathrm{l})$ and Lp-PLA2 $(-3.8 \pm 1.2 \mathrm{ng} / \mathrm{ml})$ reduction were more significant in the high-dose group $(p<0.05)$. Pearson correlation analysis showed that TC $(r=0.365)$, LDL-C $(r=0.472)$, CRP $(r=0.501)$ and ROCK1 $(r=0.675)$ were positively correlated with Lp-PLA2, while NO $(r=-0.378)$ and atorvastatin $(r=-0.511)$ were negatively correlated with Lp-PLA2.

Conclusions: Atorvastatin treatment is beneficial for reducing the LP-PLA2 level in rats with dyslipidemia, which may be related to reduced ROCK1 expression in a dose-dependent manner.

Key words: dyslipidemia, endothelial dysfunction, statins, lipoproteinassociated phospholipase A2.

\author{
Corresponding author: \\ Weiyi Mai \\ Department of Cardiology \\ The First Affiliated Hospital \\ of Sun Yat-sen University \\ 58 Zhongshan Road 2 \\ Guangzhou 510080, China \\ Phone: +86 2087755766 , \\ ext. 8164 \\ Fax: +862087755766 \\ ext. 8756 \\ E-mail:wymai@hotmail.com
}




\section{Introduction}

Dyslipidemia, mainly characterized by an increased plasma level of low-density lipoprotein cholesterol ( $L D L-C)$, is a major risk factor for atherosclerotic cardiovascular diseases (ASCVD) [1-4]. Previously, numerous studies have shown that endothelial dysfunction was the underlying mechanism contributing to dyslipidemia-mediated atherogenesis and ASCVD development [5-7]. In recent decades, some studies have revealed that an increased plasma level of lipoprotein-associated phospholipase A2 (Lp-PLA2) was also independently associated with atherosclerosis progress and ASCVD [8-11]. Briefly, Lp-PLA2 is an enzyme excreted by inflammatory cells such as macrophages which hydrolyze oxidized-LDL into lysophosphatidylcholine (Lyso-PC) and oxidized non-esterified fatty acids (oxNEFAs), both of which subsequently play critical and complex roles in atherosclerotic plaque expansion and rupture [12].

Statin is a HMG-CoA reductase inhibitor and has been broadly applied in clinical practice for dyslipidemia and ASCVD treatment. The underlying mechanisms attributing to statins' cardiovascular benefits include lowering plasma LDL-C level and anti-inflammation, endothelial protection, anti-oxidation and pro-angiogenesis $[13,14]$. However, whether statin therapy is beneficial for decreasing plasma Lp-PLA2 level is controversial and the underlying mechanisms are also not fully explored yet.

We produced a dyslipidemia rat model and used different doses of atorvastatin to evaluate the change of plasma Lp-PLA2 level. The results from our current research might help to broaden our knowledge about the effects of statins on LP-PLA2 regulation and its associated potential mechanisms.

\section{Material and methods}

\section{Dyslipidemia rat model production}

A total of 40 male Sprague-Dawley (SD) rats weighing 210-220 g were used in the present research (rats were obtained from the Experimental Animal Center of Sun Yat-sen University, Guangzhou, Guangdong, China). All rats received humane care in compliance with the Guide for the Care and Use of Laboratory Animals of the Institute of Laboratory Animal Resources, National Research Council. Among the 40 rats, 10 were randomly selected as the normal-control group with prescription of normal rat chow, and the other 30 rats were used to produce a dyslipidemia model. The protocol for dyslipidemia model production was in accordance with a previously described study with prescription of a high-fat and high-cholesterol diet including cholesterol $4.0 \%$, propylthiouracil $0.3 \%$, cholic acid $0.4 \%$, and lard $10.0 \%$ for a total of 6 weeks [15]. Thereafter, fast- ing lipid profiles were evaluated to confirm successful establishment of the dyslipidemia model. Fasting lipid profiles in the normal-control group were also measured using an auto-analyzer (Hitachi 7100, Japan) with the corresponding kits from Wako Inc (Enid, OK, USA). The plasma Lp-PLA2 level measurement kit was brought from CUSABIO BIOTECH CO., Ltd. USA (Catalog No. CSB-E08320r) and the performances were conducted in accordance to the manual instructions. In brief, the enzyme-substrate reaction was terminated by the addition of a sulfuric acid solution and the color change was measured spectrophotometrically at a wavelength of $450 \pm 2 \mathrm{~nm}$. The concentration of Lp-PLA2 in the samples was then determined by comparing the optical density of the samples to the standard curve, and the minimum detectable dose of Lp-PLA2 was typically less than $1.95 \mathrm{ng} /$ $\mathrm{ml}$. Each measurement was performed 3 times and the values were averaged.

\section{Atorvastatin treatment}

In the normal-control group, $3 \mathrm{ml}$ saline was administered by gavage in the morning around 8-10 am, and the 30 dyslipidemia rats were randomly divided into low-dose atorvastatin $(5 \mathrm{mg} / \mathrm{kg} /$ day), high-dose atorvastatin (20 mg/kg/day) and without-treatment groups ( $3 \mathrm{ml}$ saline). The doses of atorvastatin we used in the current study were based on our previous study in which we found that $5 \mathrm{mg} / \mathrm{kg} /$ day and $20 \mathrm{mg} / \mathrm{kg} /$ day were safe and efficacious for rats with dyslipidemia [16]. In brief, atorvastatin was dissolved in dimethyl-sulfoxide (DMSO, working concentration $10 \mathrm{mmol} / \mathrm{l}$ ) and was administered by gavage which was similar to our previous study [17]. The duration of treatment was 14 days and then fasting plasma was used for evaluation of lipid profiles and plasma Lp-PLA2 level.

Measurement of C-reactive protein, nitric oxide and rho-associated kinase 1 levels

At 6 weeks of high-fat and high-cholesterol diet treatment and at 14 days of atorvastatin treatment, fasting plasma was used for C-reactive protein (CRP) assessment with an enzyme linking immune-absorbent assay (ELISA) kit (Biomatik, USA, Cat. No. EKE51580); all performances were in accordance with the manual instructions, and the detection range was $0.156-10 \mathrm{ng} / \mathrm{ml}$. Nitric oxide level was evaluated by the nitrite reductase method using the Total Nitric Oxide Kit (Beyotime, Haimen, China, Cat. No. S0023). Rho-associated kinase 1 (ROCK1) level was also measured using an ELISA kit (CUSABIO, USA, Cat. No. CSB-EL020058RA) with a detection range of $125-8000 \mathrm{pg} / \mathrm{ml}$. Three independent measurements were performed and the values were averaged. 


\section{Alanine aminotransferase and creatine kinase measurement}

Plasma alanine aminotransferase (ALT) and creatine kinase (CK) levels were also measured using the Automatic Biochemistry Analyzer (Hitachi 7150, Tokyo, Japan) to evaluate whether atorvastatin treatment would cause potential side effects.

\section{Statistical analysis}

All continuous variables were expressed as mean \pm SD, and analyses were performed with SPSS software, version 17.0 (SPSS Science, Chicago, IL, USA). Statistical significance of differences between groups was evaluated using one-way ANOVA, and changes of pre- and post-atorvastatin treatment within each group were also compared using one-way ANOVA. Pearson correlation analysis was performed to evaluate the association of parameters of interest and Lp-PLA2; and a value of $p<0.05$ was considered statistically significant.

\section{Results}

\section{Assessment of dyslipidemia model establishment}

At 6 weeks of high-fat and high-cholesterol diet treatment, parameters of interest were evaluated and compared. As shown in Table I, compared with the normal-control group, fasting plasma total cholesterol (TC) and LDL-C levels were significantly increased in the dyslipidemia groups, indicating that the dyslipidemia model was successfully established. In addition, compared with the normal-control group, plasma NO levels were significantly decreased in the dyslipidemia groups with attendant elevation of plasma CRP and ROCK1 levels $(p<0.05)$.

\section{Assessment of the effects of atorvastatin treatment}

At 14 days of atorvastatin treatment, parameters of interests were evaluated and compared between different groups. As shown in Table II, compared with the without-treatment group, plasma levels of TC and LDL-C in the high-dose atorvastatin group were significantly reduced $(p<0.05)$, and there were no significant differences in TC or LDL-C between the high-dose and low-dose groups or the low-dose and without-treatment groups. Notably, compared with the low-dose and without-treatment groups, NO up-regulation and CRP and ROCK 1 reduction were more significant in the high-dose group $(p<0.05)$. Compared with without-treatment group, significant differences in NO, CRP and ROCK1 levels were also observed in the low-dose group ( $p<0.05)$. No significant elevations of ALT or CK were observed in either atorvastatin treatment group.

\section{Changes of plasma Lp-PLA2 levels}

Plasma LP-PLA2 levels were evaluated at 6 weeks of high-fat and high-cholesterol diet treatment and at 14 days of atorvastatin treatment. As shown in Figure 1, compared with the normal-control group, plasma Lp-PLA2 levels were significantly elevated in the dyslipidemia group at 6 weeks of high-fat and high-cholesterol diet treatment $(p<0.05)$. At 14 days of atorvastatin treatment, compared with the without-treatment group, the plasma Lp-PLA2 level was significantly reduced in the high-dose group ( $p<0.05)$; although plasma Lp-PLA2 level was lower in the low-dose group when compared with the without-treatment group, no statistical significance was observed $(p=0.96)$.

Table I. At 6 weeks of high-fat and high-cholesterol diet treatment ( $n=10$ each group)

\begin{tabular}{|c|c|c|c|c|}
\hline Variables & Normal - control & High-dose & Low-dose & Without treatment \\
\hline $\mathrm{TC}[\mathrm{mmol} / \mathrm{l}]$ & $4.2 \pm 0.3^{\star}$ & $5.3 \pm 0.6$ & $5.2 \pm 0.5$ & $5.3 \pm 0.5$ \\
\hline LDL-C [mmol/I] & $2.8 \pm 0.4^{\star}$ & $3.7 \pm 0.3$ & $3.7 \pm 0.4$ & $3.7 \pm 0.4$ \\
\hline $\mathrm{HDL}-\mathrm{C}[\mathrm{mmol} / \mathrm{l}]$ & $1.2 \pm 0.2$ & $1.0 \pm 0.3$ & $1.0 \pm 0.3$ & $1.0 \pm 0.2$ \\
\hline $\mathrm{TG}[\mathrm{mmol} / \mathrm{l}]$ & $1.6 \pm 0.4$ & $1.8 \pm 0.5$ & $1.7 \pm 0.5$ & $1.7 \pm 0.6$ \\
\hline CRP $[\mathrm{ng} / \mathrm{ml}]$ & $2.0 \pm 0.8^{\star}$ & $5.3 \pm 1.1$ & $5.2 \pm 1.0$ & $5.2 \pm 1.2$ \\
\hline $\mathrm{NO}[\mu \mathrm{mol} / \mathrm{ll}]$ & $10.7 \pm 1.6^{*}$ & $6.3 \pm 1.9$ & $6.2 \pm 1.4$ & $6.3 \pm 1.6$ \\
\hline ROCK1 [mmol/l] & $489 \pm 56^{\star}$ & $844 \pm 103$ & $827 \pm 124$ & $833 \pm 120$ \\
\hline $\mathrm{ALT}[\mathrm{U} / \mathrm{I}]$ & $25 \pm 8$ & $28 \pm 6$ & $26 \pm 9$ & $26 \pm 7$ \\
\hline CK $[\mathrm{U} / \mathrm{I}]$ & $10 \pm 2$ & $12 \pm 4$ & $10 \pm 4$ & $11 \pm 3$ \\
\hline
\end{tabular}


Table II. At 14 days of atorvastatin treatment ( $n=10$ each group)

\begin{tabular}{|c|c|c|c|c|}
\hline Variables & Normal - control & High-dose & Low-dose & Without treatment \\
\hline $\mathrm{TC}[\mathrm{mmol} / \mathrm{l}]$ & $4.1 \pm 0.4$ & $4.5 \pm 0.4^{*}$ & $4.8 \pm 0.5$ & $5.1 \pm 0.5$ \\
\hline LDL-C [mmol/l] & $2.8 \pm 0.5$ & $3.2 \pm 0.4^{*}$ & $3.5 \pm 0.5$ & $3.7 \pm 0.6$ \\
\hline $\mathrm{HDL}-\mathrm{C}[\mathrm{mmol} / \mathrm{l}]$ & $1.3 \pm 0.2$ & $1.1 \pm 0.3$ & $1.1 \pm 0.2$ & $1.0 \pm 0.3$ \\
\hline TG [mmol/l] & $1.7 \pm 0.3$ & $1.8 \pm 0.3$ & $1.8 \pm 0.4$ & $1.8 \pm 0.5$ \\
\hline CRP [ng/ml] & $2.2 \pm 0.6$ & $4.1 \pm 1.0^{\text {*\# }}$ & $4.8 \pm 1.1^{8}$ & $5.4 \pm 1.3$ \\
\hline $\mathrm{NO}[\mu \mathrm{mol} / \mathrm{l}]$ & $10.2 \pm 1.8$ & $8.1 \pm 1.5^{\text {*\# }}$ & $7.4 \pm 1.6^{8}$ & $6.3 \pm 1.8$ \\
\hline ROCK1 [mmol/l] & $493 \pm 67$ & $706 \pm 118^{\star \#}$ & $752 \pm 106^{\&}$ & $847 \pm 132$ \\
\hline ALT [U/l] & $24 \pm 7$ & $32 \pm 10$ & $30 \pm 9$ & $28 \pm 6$ \\
\hline CK [U/I] & $12 \pm 4$ & $15 \pm 7$ & $16 \pm 6$ & $15 \pm 8$ \\
\hline
\end{tabular}

${ }^{*} p<0.05$ vs. without-treatment group, ${ }^{*} p<0.05$ vs. low-dose group, ${ }^{\circledR} p<0.05$ vs. without-treatment group; expressed as mean \pm SD.

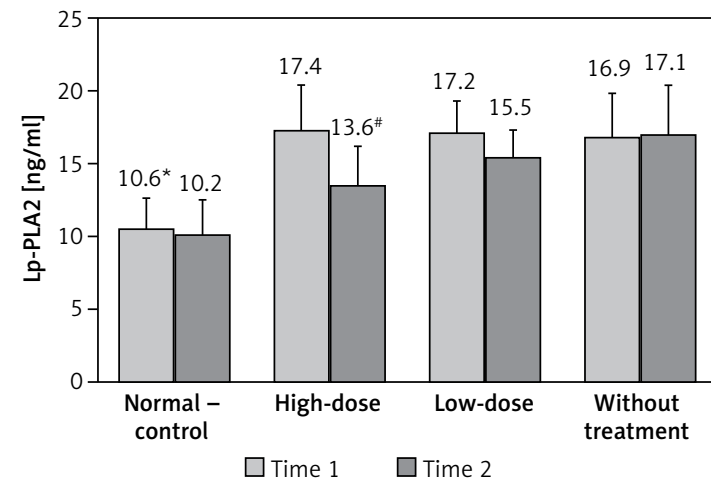

Figure 1. Changes of Lp-PLA2. Time 1 = at 6 weeks of high-fat and high-cholesterol diet treatment. Time 2 = at 14 days of atorvastatin treatment

${ }^{*} p<0.05$ vs. other groups at time $1 ;{ }^{*} p<0.05$ vs. low-dose and without-treatment groups at time 2

\section{Changes of parameters of interest before and after atorvastatin treatment within each group}

As presented in Table III, changes of parameters of interest before and after atorvastatin treatment within each group showed that the magnitude of improvement of dyslipidemia, decreased serum levels of CRP, ROCK1 and Lp-PLA2, and increased serum level of NO were most prominent in the high-dose group when compared to the low-dose and without-treatment groups $(p<0.05)$. Similarly, compared to the without-treatment group, the changes of the above parameters were also significantly greater in the low-dose group when compared to the without-treatment group $(p<0.05)$.

\section{Pearson correlation analysis}

Pearson correlation analysis was performed to evaluate the association of plasma Lp-PLA2 level and parameters of interest. As shown in Table IV, TC, LDL-C, CRP and ROCK1 were positively correlated with Lp-PLA2, while NO and statins both were negatively correlated with Lp-PLA2. Notably, the correlation coefficient of ROCK1 was the largest.

\section{Discussion}

Our preliminary research shows that in rats with 6 weeks of high-fat and high-cholesterol diet treatment, dyslipidemia establishment is accom-

Table III. Changes of parameters of interest before and after atorvastatin treatment within each group $(n=10$ each group)

\begin{tabular}{|lcccc|}
\hline Variables & Normal - control & High-dose & Low-dose & Without treatment \\
\hline TC $[\mathrm{mmol} / \mathrm{l}]$ & $-0.2 \pm 0.1$ & $-0.8 \pm 0.3^{*}$ & $-0.4 \pm 0.2$ & $-0.2 \pm 0.1$ \\
\hline LDL-C $[\mathrm{mmol} / \mathrm{l}]$ & $0.2 \pm 0.1$ & $-0.5 \pm 0.3^{*}$ & $-0.2 \pm 0.1$ & $-0.1 \pm 0.1$ \\
\hline HDL-C $[\mathrm{mmol} / \mathrm{l}]$ & $0.2 \pm 0.1$ & $0.2 \pm 0.1$ & $0.1 \pm 0.1$ & $0.1 \pm 0.1$ \\
\hline TG $[\mathrm{mmol} / \mathrm{l}]$ & $0.2 \pm 0.1$ & $0.1 \pm 0.1$ & $0.2 \pm 0.1$ & $0.2 \pm 0.1$ \\
\hline CRP $[\mathrm{ng} / \mathrm{ml}]$ & $0.2 \pm 0.1$ & $-0.8 \pm 0.4^{* \#}$ & $-0.4 \pm 0.2^{8}$ & $0.2 \pm 0.1$ \\
\hline NO $[\mu \mathrm{mol} / \mathrm{l}]$ & $-0.5 \pm 0.3$ & $1.8 \pm 1.1^{* \#}$ & $1.2 \pm 0.6^{\&}$ & $0.2 \pm 0.2$ \\
\hline ROCK1 $[\mathrm{mmol} / \mathrm{l}]$ & $11 \pm 8$ & $-124 \pm 65^{* \#}$ & $-72 \pm 33^{\&}$ & $14 \pm 7$ \\
\hline ALT $[\mathrm{U} / \mathrm{l}]$ & $-2 \pm 1$ & $4 \pm 2$ & $4 \pm 2$ & $2 \pm 1$ \\
\hline CK $[\mathrm{U} / \mathrm{l}]$ & $2 \pm 1$ & $3 \pm 2$ & $6 \pm 3$ & $4 \pm 3$ \\
\hline Lp-PLA2 $[\mathrm{ng} / \mathrm{ml}]$ & $-0.4 \pm 0.2$ & $-3.8 \pm 1.2^{* \#}$ & $-1.7 \pm 0.9^{\&}$ & $0.3 \pm 0.2$ \\
\hline
\end{tabular}

${ }^{*} p<0.05$ vs. without-treatment group; ${ }^{*} p<0.05$ vs. low-dose group; ${ }^{\star} p<0.05$ vs. without-treatment group; expressed as mean \pm SD. 
panied by endothelial dysfunction, and increased plasma levels of inflammatory cytokine, Lp-PLA2 and ROCK1. In addition, the Pearson correlation analysis reveals that plasma LP-PLA2 level is positively correlated with serum cholesterol, CRP and ROCK1 levels and negatively correlated with serum NO level and statins therapy. These data indicate that dyslipidemia may be related to LP-PLA2 elevation and atorvastatin treatment is beneficial for reducing serum Lp-PLA2 level in a dose-dependent manner.

Dyslipidemia is a major risk factor for ASCVD, and the mechanism is mediated by dyslipidemia induced-inflammation and endothelial dysfunction [18]. For example, Huang et al. [15] reported that in rats with dyslipidemia, using atorvastatin and colchicine therapy was better than atorvastatin alone for improving endothelial dysfunction, and these benefits were attributed to the robust effects of colchicine on inflammation amelioration. Consistent with the previous study, our study also showed that atorvastatin treatment could improve dyslipidemia as well as endothelial dysfunction. However, in our study, we found that 14 days of low-dose atorvastatin ( $5 \mathrm{mg} / \mathrm{kg} /$ day) treatment did not show significant improvement of endothelial dysfunction and dyslipidemia as compared to the without-treatment group. The benefits were most prominent in the high-dose atorvastatin group (20 mg/kg/day) and in Huang's research using $10 \mathrm{mg} / \mathrm{kg} /$ day, suggesting that the effect of short-term atorvastatin therapy on endothelial protection was dose-dependent.

The mechanisms underlying the endothelial protection of atorvastatin therapy are multiple, and among them, suppressing ROCK1 expression is one of the intensively investigated fields [19]. ROCK 1 is a kinase which regulates multiple cellular functions such as decreasing endothelial nitric oxide synthase (eNOS) expression, promoting macrophage chemotaxis and migration, and activating platelets [20, 21]. Previous studies have showed that ROCK1 elevation reduced nitric oxide production by means of inhibiting eNOS expression and activity $[22,23]$. In our current research, we also found that dyslipidemia contributed to ROCK1 elevation, and based on previous reports we speculated that ROCK1 elevation resulted in endothelial dysfunction and atorvastatin treatment suppressing ROCK1 expression. Indeed, our results showed that (as presented in Table II), compared to the without-treatment group, plasma ROCK1 levels in both high-dose and low-dose atorvastatin treatment groups were attenuated, although statistical significance was only observed in the high-dose group.

Whether statin therapy could reduce Lp-PLA2 level is uncertain. Lp-PLA2 is an enzyme excreted by inflammatory cells such as macrophages
Table IV. Pearson correlation analysis

\begin{tabular}{|lcc|}
\hline Variables & Correlation coefficient & $P$-value \\
\hline TC $[\mathrm{mmol} / \mathrm{l}]$ & 0.365 & 0.017 \\
\hline LDL-C $[\mathrm{mmol} / \mathrm{l}]$ & 0.472 & 0.003 \\
\hline HDL-C $[\mathrm{mmol} / \mathrm{l}]$ & -0.126 & 0.064 \\
\hline TG $[\mathrm{mmol} / \mathrm{l}]$ & 0.108 & 0.142 \\
\hline CRP $[\mathrm{ng} / \mathrm{ml}]$ & 0.501 & $<0.001$ \\
\hline NO $[\mu \mathrm{mol} / \mathrm{l}]$ & -0.378 & 0.006 \\
\hline ROCK1 $[\mathrm{mmol} / \mathrm{l}]$ & 0.675 & $<0.001$ \\
\hline Statin usage & -0.511 & $<0.001$ \\
\hline
\end{tabular}

which then hydrolyzes ox-LDL into two more potent pro-atherosclerotic metabolites, Lyso-PC and oxNEFAs. It is therefore conceivable that reducing the Lp-PLA2 level would contribute to deceleration of atherosclerosis progression and reduction of cardiovascular events. Statins have pleiotropic effects such as decreasing macrophage infiltration and anti-oxidation, and it is biologically plausible that statin therapy could reduce LP-PLA2 excretion from macrophages. For example, White et al. [24] reported that Lp-PLA2 attenuation by pravastatin therapy during the first year was significantly associated with reduced ASCVD events, which was independent of LDL-C change. Nevertheless, in another study, Albert et al. [25] found that after adjustment for LDL-C, the effect of pravastatin on Lp-PLA2 reduction was no longer statistically significant. Our current experimental study showed that atorvastatin therapy could reduce Lp-PLA2 in a dose-dependent manner, which was also further supported by the strong correlation coefficient of -0.511 as presented in Table IV. In addition, as evident in the Pearson correlation analysis, the positive relationship was strongest between Lp-PLA2 and ROCK1. In light of the pathophysiological effects of ROCK1 and the effects of statins on ROCK1 expression [26, 27], we speculated that the efficacies of atorvastatin treatment on LpPLA2 attenuation might be attributed to ROCK1 reduction. Indeed, ROCK1 reduction leads to a macrophage migration decrease which in turn could lead to diminished Lp-PLA2 production. In addition, endothelial function improvement by attenuated ROCK 1 could also decrease macrophage infiltration and accumulation, which in turn further attenuate LP-PLA2 production.

In conclusion, our present research reveals that atorvastatin treatment is beneficial for reducing plasma LP-PLA2 level in rats with dyslipidemia and the underlying mechanism may be related to attenuation of ROCK 1 expression in a dose-dependent manner. The clinical implication of our present research is that even short-term dyslipidemia 
could result in severe impairment of endothelial function and high-dose atorvastatin prescription may result in prompt storage of endothelial function, which in turn may help to prevent atherosclerosis progression.

\section{Acknowledgments}

Dongdan Zheng and Anping Cai contributed equally to this work.

The present study is supported by the Guangdong Natural Foundation of Science (2016A0303 13794) and the Technology Project of Guangdong Province (2011B031800021).

\section{Conflict of interest}

The authors declare no conflict of interest.

\section{References}

1. Johnson ML, Pietz K, Battleman DS, Beyth RJ. Prevalence of comorbid hypertension and dyslipidemia and associated cardiovascular disease. Am J Manag Care 2004; 10: 926-32.

2. Mahdavi H, Kim JB, Safarpour S, Tien DA, Navab M. Dyslipidemia and cardiovascular diseases. Curr Opin Lipidol 2009; 20: 157-8.

3. Siri-Tarino PW, Krauss RM. Diet, lipids, and cardiovascular disease. Curr Opin Lipidol 2016; 27: 323-8.

4. Cheng HG, Patel BS, Martin SS, et al. Effect of comprehensive cardiovascular disease risk management on longitudinal changes in carotid artery intima-media thickness in a community-based prevention clinic. Arch Med Sci 2016; 12: 728-35.

5. von SBJ, Reinhard H, Hansen TW, et al. Markers of inflammation and endothelial dysfunction are associated with incident cardiovascular disease, all-cause mortality, and progression of coronary calcification in type 2 diabetic patients with microalbuminuria. J Diabetes Complications 2016; 30: 248-55.

6. Cai H, Harrison DG. Endothelial dysfunction in cardiovascular diseases: the role of oxidant stress. Circ Res 2000; 87: 840-4.

7. Heitzer T, Schlinzig T, Krohn K, Meinertz T, Münzel T. Endothelial dysfunction, oxidative stress, and risk of cardiovascular events in patients with coronary artery disease. Circulation 2001; 104: 2673-8.

8. Vittos O, Toana B, Vittos A, Moldoveanu E. Lipoprotein-associated phospholipase A2 (Lp-PLA2): a review of its role and significance as a cardiovascular biomarker. Biomarkers 2012; 17: 289-302.

9. Sudhir K. Clinical review: Lipoprotein-associated phospholipase A2, a novel inflammatory biomarker and independent risk predictor for cardiovascular disease. J Clin Endocrinol Metab 2005; 90: 3100-5.

10. Zheng D, Zeng F, Cai A, et al. Baseline elevated Lp-PLA2 is associated with increased risk for re-stenosis after stent placement. Lipids Health Dis 2014; 13: 41.

11. Colley KJ, Wolfert RL, Cobble ME. Lipoprotein associated phospholipase $A(2)$ : role in atherosclerosis and utility as a biomarker for cardiovascular risk. EPMA J 2011; 2: 27-38.

12. Wilensky RL, Macphee CH. Lipoprotein-associated phospholipase A(2) and atherosclerosis. Curr Opin Lipidol 2009; 20: 415-20.
13. Wang CY, Liu PY, Liao JK. Pleiotropic effects of statin therapy: molecular mechanisms and clinical results. Trends Mol Med 2008; 14: 37-44.

14. Epstein M, Campese VM. Pleiotropic effects of 3-hydroxy3-methylglutaryl coenzyme a reductase inhibitors on renal function. Am J Kidney Dis 2005; 45: 2-14.

15. Huang C, Cen C, Wang C, Zhan H, Ding X. Synergistic effects of colchicine combined with atorvastatin in rats with hyperlipidemia. Lipids Health Dis 2014; 13: 67.

16. Zheng D, Liang $\mathrm{Q}$, Zeng $\mathrm{F}$, et al. Atorvastatin protects endothelium by decreasing asymmetric dimethylarginine in dyslipidemia rats. Lipids Health Dis 2015; 14: 41.

17. Cai A, Qiu R, Li L, et al. Atorvastatin treatment of rats with ischemia-reperfusion injury improves adiposederived mesenchymal stem cell migration and survival via the SDF-1 $\alpha /$ CXCR-4 axis. PLoS One 2013; 8: e79100.

18. Ghomari-Boukhatem H, Bouchouicha A, Mekki K, Chenni K, Belhadj M, Bouchenak M. Blood pressure, dyslipidemia and inflammatory factors are related to body mass index in scholar adolescents. Arch Med Sci 2017; 13: 46-52.

19. Sawada N, Liao JK. Rho/Rho-associated coiled-coil forming kinase pathway as therapeutic targets for statins in atherosclerosis. Antioxid Redox Signal 2014; 20: 1251-67.

20. Cai A, Li L, Zhou Y. Pathophysiological effects of RhoA and Rho-associated kinase on cardiovascular system. J Hypertens 2016; 34: 3-10.

21. Jasińska-Stroschein M, Owczarek J, Sołtysiak U, OrszulakMichalak D. Rosuvastatin intensifies the beneficial effects of rho-kinase inhibitor in reversal of monocrotaline-induced pulmonary hypertension. Arch Med Sci 2016; 12: 898-905.

22. Rikitake Y, Liao JK. Rho GTPases, statins, and nitric oxide. Circ Res 2005; 97: 1232-5.

23. Soga J, Noma K, Hata T, et al. Rho-associated kinase activity, endothelial function, and cardiovascular risk factors. Arterioscler Thromb Vasc Biol 2011; 31: 2353-9.

24. White HD, Simes J, Stewart RA, et al. Changes in lipoprotein-associated phospholipase A2 activity predict coronary events and partly account for the treatment effect of pravastatin: results from the Long-Term Intervention with Pravastatin in Ischemic Disease study. J Am Heart Assoc 2013; 2: e000360.

25. Albert MA, Glynn RJ, Wolfert RL, Ridker PM. The effect of statin therapy on lipoprotein associated phospholipase A2 levels. Atherosclerosis 2005; 182: 193-8.

26. Nohria A, Prsic A, Liu PY, et al. Statins inhibit Rho kinase activity in patients with atherosclerosis. Atherosclerosis 2009; 205: 517-21.

27. Garg PK, McClelland RL, Jenny NS, et al. Association of lipoprotein-associated phospholipase $\mathrm{A}(2)$ and endothelial function in the Multi-Ethnic Study of Atherosclerosis (MESA). Vasc Med 2011; 16: 247-52. 